Recepción: 31 / 10 / 2018

Aceptación: 18 / $12 / 2018$

Publicación: 20 / 01 / 2019

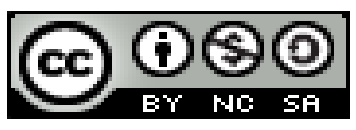

Ciencias de la computación

Artículo original

\title{
Las tecnologías de la información y la comunicación en el aula universitaria
}

\author{
Information and communication technologies in the university \\ classroom
}

Tecnologias de informação e comunicação na sala de aula da universidade

\author{
Alexandra O. Pazmiño-Armijos ${ }^{\mathrm{I}}$ \\ apazmino_a@espoch.edu.ec \\ Jairo R. Jácome-Tinoco II \\ jjacome@espoch.edu.ec \\ Paulina F. Bolaños-Logroño III \\ paulina.bolanos@espoch.edu.ec \\ Gloria E. Miño-Cascante ${ }^{\mathrm{IV}}$ \\ gmino@espoch.edu.ec
}

Correspondencia: apazmino_a@espoch.edu.ec

\footnotetext{
I Magíster en Informática Empresarial, Especialista en Redes de Comunicación de Datos, Ingeniera en Electrónica y Computación, tecnólogo en Informática Aplicada, Docente de la Escuela Politécnica de Chimborazo ESPOCH, Riobamba, Ecuador.

${ }^{\text {II }}$ Magíster en Sistemas de Telecomunicaciones, Ingeniero en Electrónica y Computación, Tecnólogo en Informática Aplicada, Docente de la Escuela Politécnica de Chimborazo ESPOCH, Riobamba, Ecuador.

III Magíster en Sistemas de Control y Automatización Industrial, Ingeniera en Electrónica y Computación, Tecnóloga en Informática Aplicada, Docente de la Escuela Politécnica de Chimborazo ESPOCH, Riobamba, Ecuador.

IV Magíster en Dirección de Empresas Mención Proyectos, Magíster en Docencia Universitaria e Investigación Educativa, Doctor en Ciencias Económicas, Ingeniero de Empresas, Docente de la Escuela Politécnica de Chimborazo ESPOCH, Riobamba, Ecuador.
} 


\title{
Resumen
}

La transformación digital y la aplicación de la tecnología en las aulas, se han convertido en elementos importantes de las TIC durante la formación universitaria, constituyéndose en una de las innovaciones a considerar en la educación en esta era de la tecnología. El presente artículo tiene como objetivo caracterizar el uso y manejo de las TIC's en el ámbito educativo universitario. La metodología fue de tipo descriptiva-analítica y con un diseño no experimental de campo. Para la obtención de la información se utilizó como instrumento una lista de cotejo y como técnica la encuesta. La población la constituyeron 135 estudiantes de educación universitaria. Entre sus resultados se pudo constatar que el $92 \%$ de los estudiantes utilizan diariamente la computadora para apoyarse en las labores propias de sus estudios y el $98 \%$ manejan y tienen una cuenta de correo que visitan de forma muy frecuente, permitiendo entre sus conclusiones indicar que existe una alta dedicación horaria de navegación a través de Internet Explorer por parte de los estudiantes, específicamente utilizando el buscador google, seguido del Yahoo y Bing.

Palabras clave: tecnologías; información; educación y formación universitaria.

\begin{abstract}
Digital transformation and the application of technology in the classroom have become important elements of ICT during university training, becoming one of the innovations to be considered in education in this era of technology. The objective of this article is to characterize the use and management of ICTs in the university educational environment. The methodology was descriptive-analytical and with a non-experimental field design. To obtain the information, a checklist was used as a tool and the survey as a technique. The population was made up of 135 university education students. Among its results it was found that $92 \%$ of students use the computer daily to support themselves in the work of their studies and $98 \%$ manage and have a mail account that they visit very frequently, allowing among their conclusions to indicate that there is a high hourly dedication of browsing through Internet Explorer by students, specifically using the Google search engine, followed by Yahoo and Bing.
\end{abstract}

Keywords: technologies; information; education and university education. 


\section{Resumo}

A transformação digital e a aplicação da tecnologia na sala de aula se tornaram elementos importantes das TIC durante o treinamento universitário, tornando-se uma das inovações a serem consideradas na educação nesta era da tecnologia. O objetivo deste artigo é caracterizar o uso e o gerenciamento das TICs no ambiente educacional universitário. A metodologia foi descritivoanalítica e com delineamento de campo não experimental. Para obter as informações, uma lista de verificação foi usada como uma ferramenta e a pesquisa como uma técnica. A população era composta por 135 estudantes universitários. Entre seus resultados, verificou-se que 92\% dos alunos utilizam o computador diariamente para se sustentar no trabalho de seus estudos e $98 \%$ gerenciam e possuem uma conta de e-mail que visitam com muita frequência, permitindo entre suas conclusões indicar que Há uma alta dedicação por hora de navegar pelo Internet Explorer pelos alunos, usando especificamente o mecanismo de busca do Google, seguido pelo Yahoo e pelo Bing.

Palavras chave: tecnologias; informação; educação e ensino universitário.

\section{Introducción}

Las tecnologías de la información y la comunicación (TIC), en el ámbito educativo contribuyen a la transformación de la educación. En este sentido desde la UNESCO (2016), se presta ayuda a los países en materia de tecnología con la finalidad de lograr el acceso universal a la educación, reducir las diferencias en el aprendizaje, apoyar el desarrollo de los docentes, mejorar la calidad y la pertinencia del aprendizaje, reforzar la integración y perfeccionar la gestión y administración de la educación. Asimismo, propone actividades de fomento de la capacidad, asesoramiento técnico, publicaciones, investigaciones y conferencias internacionales como las Conferencias Internacionales sobre las TIC y la educación después de 2015 o la Semana del Aprendizaje Mediante Dispositivos Móviles, como ayuda a los gobiernos y a otras partes interesadas a valerse de las tecnologías para fomentar el aprendizaje.

Todo lo expuesto, según el proyecto innovador en Croacia de escuelas digitalmente maduras (2018), responde a una cuarta revolución industrial, que ha modificado profundamente numerosos aspectos de nuestras vidas, fundamentalmente nuestra manera de interactuar, aprender y trabajar. En consecuencia, el uso racional de las tecnologías en la educación responde a los 
cambios actuales y futuros de nuestra sociedad. Esto estimula la participación activa de los alumnos en el proceso de aprendizaje, y contrariamente a la enseñanza tradicional facilita la reflexión crítica, la solución de los problemas y la colaboración, así como un mejor acceso a la información, una mejor visualización de los contenidos, una adaptación más fácil a las necesidades específicas de los alumnos, etc.

También, exponen que las instituciones maduras en el ámbito digital y la utilización adecuada de las TIC facilitarán la transparencia y la eficacia en la administración de las escuelas, y fundamentalmente el desarrollo de las competencias digitales y la preparación de los docentes para que adopten enfoques más innovadores en su trabajo con los estudiantes. Por otra parte, los estudiantes que dominan las tecnologías digitales se hallarán en mejores condiciones de continuar sus estudios y serán más competitivos en el mercado laboral.

Cabe destacar que las redes sociales, actualmente participan en el diario vivir de un alto porcentaje de las personas, específicamente entre los usuarios de Internet y de telefonía. Toca, G. (2018), en la revista Ekos público que la cantidad de usuarios ecuatorianos en Internet, asciende a un promedio de 13,5 millones. Mientras que el promedio de usuarios móviles es del 92\%. Además, proporciona el número de usuarios en las distintas redes sociales y hace un recuento de las páginas web más visitadas en el país. Las plataformas sociales encabezan la lista de preferencias. Siendo Facebook la numero 1 y Youtube la segunda; mientras que Instagram se posiciona en la posición 6, Twitter en la $11 \mathrm{y}$ Whastapp en el puesto en el puesto 13. Esto es evidencia que las redes sociales tienen una fuerte presencia en la vida de los ecuatorianos y tienen interacción con ellos varias veces en su día a día. En atención a lo expuesto, en este artículo se caracterizaron el uso y manejo de las TIC's en el ámbito educativo universitario.

\section{Desarrollo}

Las tecnologías de la comunicación y la información, explican Dussel y Quevedo (2010), ayudan el intercambio internacional de experiencia, permitiendo restar barreras y distancias geográficas, así como enriquecer el aprendizaje individual y colectivo logrando relaciones de amistad, parentesco, intereses comunes o compartir conocimientos. Entendiendo que las redes sociales son un medio de comunicación social, que por el contrario de los medios tradicionales que suelen ser plataformas limitadas en cuanto a la cantidad de gente que puede interactuar al mismo tiempo, 
en el caso de las redes sociales son sitios cuyo contenido es diariamente publicado por millones de personas diferentes y de fácil manejo, hasta lograr que la gente pueda recuperar o mantener el contacto con antiguos compañeros del colegio, instituto, universidad, etcétera.

Las redes sociales. Según Royero (2007) es "el conjunto de personas, comunidades, entes u organizaciones que producen, reciben e intercambian bienes o servicios sociales para su sostenimiento en un esquema de desarrollo y bienestar esperado. Dicho bienestar es mediatizado por los avances en el campo de la ciencia y la tecnología producidos y ofrecidos en su valor social y mercantil a las personas o grupos de ellas, en un territorio y en unas condiciones económicas sociales determinadas. Estos intercambios se dan a nivel local regional, nacional, internacional y global" (p,65). Se inicia la aparición de Redes como Friendster, posteriormente en el 2003 aparecen otros sitios como Tribe.net, MySpace, Ecademy, Soflow y LinkedIn. Desde entonces diversas redes se han creado, unas permanecen y otras han desaparecido. Un poco antes del 2009 hasta la actualidad, los principales competidores a nivel mundial son: Facebook, Twitter, Google+, Instagram, Pinterest, entre otras.

No obstante, las redes sociales también pueden ser consideradas como el uso de software y hardware en el entorno de Internet, y en el caso de la educación se habla del e-learning como un proceso de enseñanza-aprendizaje que se lleva a cabo a través de Internet, caracterizados por una separación física entre profesorado y estudiantes, pero con el predominio de una comunicación tanto síncrona como asíncrona, a través de la cual se lleva a cabo una interacción didáctica continuada. Además, el estudiante pasa a ser el centro de la formación, al tener que autogestionar su aprendizaje, con ayuda de tutores y compañeros. Por otro lado, el uso de las tecnologías de Internet está fundamentado en tres criterios:

-El e-learning trabaja en red, lo que lo hace capaz de ser instantáneamente actualizado, almacenado, recuperado, distribuido, y permite compartir instrucción o información.

-Es entregado al usuario final a través del uso de ordenadores utilizando tecnología estándar de Internet.

-Se enfoca en la visión más amplia del aprendizaje que va más allá de los paradigmas tradicionales de capacitación. 
Desde la perspectiva que ofrece la experiencia en el desarrollo y explotación de plataformas elearning, García Peñalvo (2001), la define como: la "capacitación no presencial que, a través de plataformas tecnológicas, posibilita y flexibiliza el acceso y el tiempo en el proceso de enseñanza-aprendizaje, adecuándolos a las habilidades, necesidades y disponibilidades de cada discente, además de garantizar ambientes de aprendizaje colaborativos mediante el uso de herramientas de comunicación síncrona y asíncrona, potenciando en suma el proceso de gestión basado en competencias." (p.5). Es importante indicar que esta herramienta puede ayudar a los usuarios no solo a aprender conceptos nuevos sino también a afianzar conocimientos y habilidades, aumentado así la autonomía y la motivación de los estudiantes por diferentes temas.

\section{Principales redes sociales en el mundo}

El informe "Social Media arround the World"elaborado entre diciembre de 2009 y enero 2010, por la empresa consultorabelga InSitesConsultingen siete regiones del mundo, donde se incluyeron 14 países (Bélgica, Holanda, Inglaterra, España, Italia, Portugal, Francia, Alemania, Rumania, EstadosUnidos, Brasil, Australia, Rusia y China), desvela que el 72\% de los usuarios de Internet pertenece al menos a una red social, lo que se traduce en que hay 940 millones de usuarios en todo el mundo. El envío de mensajes, las conversaciones en la página de inicio (en la función llamada muro) y la incorporación a páginas y/o grupos, son las actividades realizadas en las redes sociales más destacadas por los usuarios. El número medio de redes sociales utilizadas por los usuarios es de dos.

Asimismo, es importante señalar las 10 redes sociales más utilizadas por los usuarios, se trata de redes sociales directas basadas en perfiles tanto personales como profesionales. La red social más conocida en el mundo con un 51\% de usuarios de Internet es el Facebook, siendo una red social directa de perfil personal y es empleada de forma preferente en los países de habla anglosajona. Según datos publicados les siguen porcentualmente en el siguiente orden; myspace $20 \%$. twitter $17 \%$. orkut $15 \%$, hi5 $11 \%$, linkedln $9 \%$, netlog $8 \%$, xing $3 \%$, ning $2 \%$ y hyves con el $1 \%$. El mismo informe, especifica a cada uno de ellos de la siguiente forma:

Facebook. El área principal de trabajo en Facebook es el perfil, desde el que se puede ir configurando y añadiendo toda la información. Además de perfiles, existen grupos y páginas. Los perfiles y grupos están diseñados para personas físicas (éstas agregan contactos), mientras que las 
páginas son para las empresas o productos, (éstas agregan fans). Los grupos están diseñados para grupos de personas que tienen un interés común no teniendo por qué ser contactos entre ellos. Los perfiles tienen un límite de 5.000 contactos, mientras que las páginas no tienen límite de contactos y están indexadas por los buscadores. Se puede crear tres tipos de grupos: abierto (cualquier persona puede entrar), cerrado (el administrador decide quién puede entrar),y secreto(solo conocen el grupo los miembros e invitados).Entre ellos se pueden mandar mensajes privados o escribir en el muro.

YouTube. Su objetivo consiste en que el usuario descubra y dé forma al mundo a través del vídeo, declara el sitio como lema principal. Parte del objetivo de YouTube es ampliar su alcance más allá de los navegadores de Internet y permitir a los usuarios descubrir y compartir contenidos en vídeo, por ello está constantemente innovando, mejorando sus herramientas y sus API (interfaz de programación de aplicaciones). Esto incluye el acceso total a su extensa biblioteca de vídeos, una audiencia mundial y la infraestructura de transmisión y alojamiento con la que cuenta el sitio. En síntesis, lo que ofrece a los usuarios es otra forma más de unirse a una comunidad mundial de vídeos y poder participar de forma activa en su comunidad desde cualquier lugar y en cualquier momento. Los usuarios pueden insertar vídeos de YouTube en cuentas de MySpace y Facebook, blogs y otros sitios web donde cualquier persona pueda verlos. Pueden elegir entre emitir sus vídeos de forma pública o compartirlos de forma privada con sus amigos o familiares una vez subidos, realizar un seguimiento de los nuevos vídeos de sus usuarios favoritos y grabar de forma instantánea en el sitio vídeos normales o respuestas en vídeo en vez de tener que realizar la grabación primero y subir después los vídeos.

Wikipedia. Es un wiki, un medio de colaboración abierto cuyo objetivo es crear fuentes de información de forma gratuita. Se trata del proyecto más amplio de la Fundación Wikimedia. Cualquiera puede crear, modificar, completar, borrar y discutir el contenido existente, pero solo un número limitado de administradores tienen permisos para poder solucionar disputas o bloquear páginas en caso necesario. Estos administradores trabajan de manera voluntaria, ya que no reciben ninguna remuneración por su trabajo. Wikipedia ha sido creada en su totalidad por voluntarios, aunque acepta donaciones de usuarios. Se permite la utilización de toda la información contenida en la base de datos de forma gratuita. 
hi5. Es una red para hacer amigos, conocer personas y buscar pareja. Para acceder se debe crear una cuenta, donde el usuario puede configurar sus preferencias, para que así su red de amigos pueda informarse de sus gustos. También se puede jugar dentro de la red. Poseen una plataforma con varios juegos, lo que permite jugar online de manera interactiva con millones de usuarios. Cuenta con juegos localizados, bienes virtuales y su propia moneda hi5, que admite más de 60 métodos de pago, en más de 30 tipos de monedas del mundo. Existen dos secciones de datos, una para difundir la información personal del usuario: estado civil, edad, religión, idiomas, ciudad natal, y la otra el bloque o sección, que es para datos relacionados con los gustos personales: acerca de mí, música favorita, películas favoritas, libros favoritos, programa de TV favoritos, cita favorita. Todos los usuarios que acceden a una cuenta podrán ver toda la información que se haya registrado, siempre y cuando el dueño de esa no haya restringido el acceso a la misma.

MySpace. Es un sitio de interacción social centrado en crear una experiencia personalizada para sus usuarios. Esta red social está principalmente centrada en promover a aquellos usuarios que son más activos en cuanto a descubrimiento e intercambio de contenidos se refiere. Principalmente existen dos tipos de perfiles los fans y los líderes.

Meetic. Es el principal servicio de online dating o búsqueda de pareja y amistad a través de Internet. Permite realizar una búsqueda precisa de la persona que se desea, en función de la edad, origen, lugar de residencia, gustos, carácter, aficiones, físico, profesión, etc. También ofrece un chat, así como mensajería privada. Para participar, el usuario se debe dar de alta y contestar a una serie de preguntas personales como: si tiene hijos, estado civil, aspecto físico, altura, complexión, o si fuma o no. También se puede agregar una foto y un texto introductorio. Se debe validar un correo electrónico y pagar un pase para poder interactuar con los demás usuarios.

LinkedIn. Es una red social orientada a los negocios y el trabajo. Su objetivo es conectar a profesionales de todo el mundo. Para ello se debe crear un perfil que resuma la experiencia y logros profesionales. Posteriormente se pueden establecer conexiones con los contactos que uno haga y los contactos de los contactos. A través de la red se puede gestionar la información sobre uno mismo como profesional, y hacer contactos con empresas o personas. El perfil puede ser público o no. 
Xing. Es una red social de contactos profesionales. Los profesionales emplean Xing para buscar y encontrar contactos de utilidad, información relevante, nuevos negocios, nuevos candidatos, ofertas de trabajo, clientes y nuevas ideas. Cuentan con una red de contactos de 10,8 millones de usuarios y está disponible en 16 idiomas con contactos en todas las áreas y sectores profesionales. Esta red social de perfil profesional se concibe para que sus usuarios no sólo puedan interaccionar a nivel profesional, sino que también sea una herramienta útil para la planificación personal de una adecuada carrera profesional. Al estar centrada en perfiles profesionales facilita el desarrollo y establecimiento de contactos de interés para sus usuarios. Se llevan también a cabo actividades y eventos en tiempo real que complementan el trabajo en red.

Menéame. Es una web que permite enviar una historia y/o noticia que puede ser revisada por todos, promovida, o no, a la página principal. Cuando un usuario envía una noticia ésta queda en la cola de pendientes hasta que reúne los votos suficientes para ser promovida a la página principal, aunque con un claro control por parte de la web. Fue creada por Ricardo Galli, profesor del departamento de informática de la Universidad de las Islas Baleares, y Benjamí Villoslada, que colabora en todo lo que respecta al aspecto e imagen del sitio web y asuntos legales o financieros. La idea tiene su base en un digg, un sitio web que trata principalmente sobre noticias y que combina marcadores sociales, blogging y sindicación con un sistema de publicación sin editores, lo cual permite que se publiquen artículos sobre una gran variedad de géneros. Los contenidos de las noticias son libres, pero están sujetas a la revisión de los lectores que las votarán o no.

Al respecto, Zelaya (2007), considera las distintas redes sociales, como un desafío para la educación, dado el rol de formador que les corresponde, e indica que es necesario un cambio de los métodos tradicionales o convencionales por métodos innovadores para la formación abierta y continua que se ajuste a las necesidades individuales y al avance tecnológico y no a la rutina de la institución educativa.

\section{Metodología}

La investigación fue de tipo descriptiva-analítica y con un diseño no experimental de campo (Hernández et al., 2014). El corpus teórico del estudio estuvo constituido por los elementos que estructuran las TIC's en el ámbito educativo. Para la obtención de la información se utilizó como 
instrumento una lista de cotejo y como técnica la encuesta. La población la constituyeron 135 estudiantes de educación universitaria. El análisis descriptivo frecuencial se realizó considerando el paquete estadístico SPSS versión 24 .

\section{Resultados}

El resultado de la investigación se presenta a través de la información obtenida del instrumento aplicado a los investigados, las mismas se presentan en tablas y gráficos con sus respectivos análisis.

\section{Tabla1}

Análisis descriptivo frecuencial del uso computacional y acceso a internet

\begin{tabular}{ccc}
\hline ANÁLISIS/ ALTERNATIVAS & $\boldsymbol{F R}$ & $\mathbf{\%}$ \\
\hline Uso diario de la computadora & 124 & 92 \\
\hline Internet en el Hogar & 116 & 86 \\
\hline Internet en el Teléfono & 116 & 86 \\
\hline Internet en la Universidad & 46 & 34 \\
\hline
\end{tabular}

Fuente: Elaboración propia (2018).

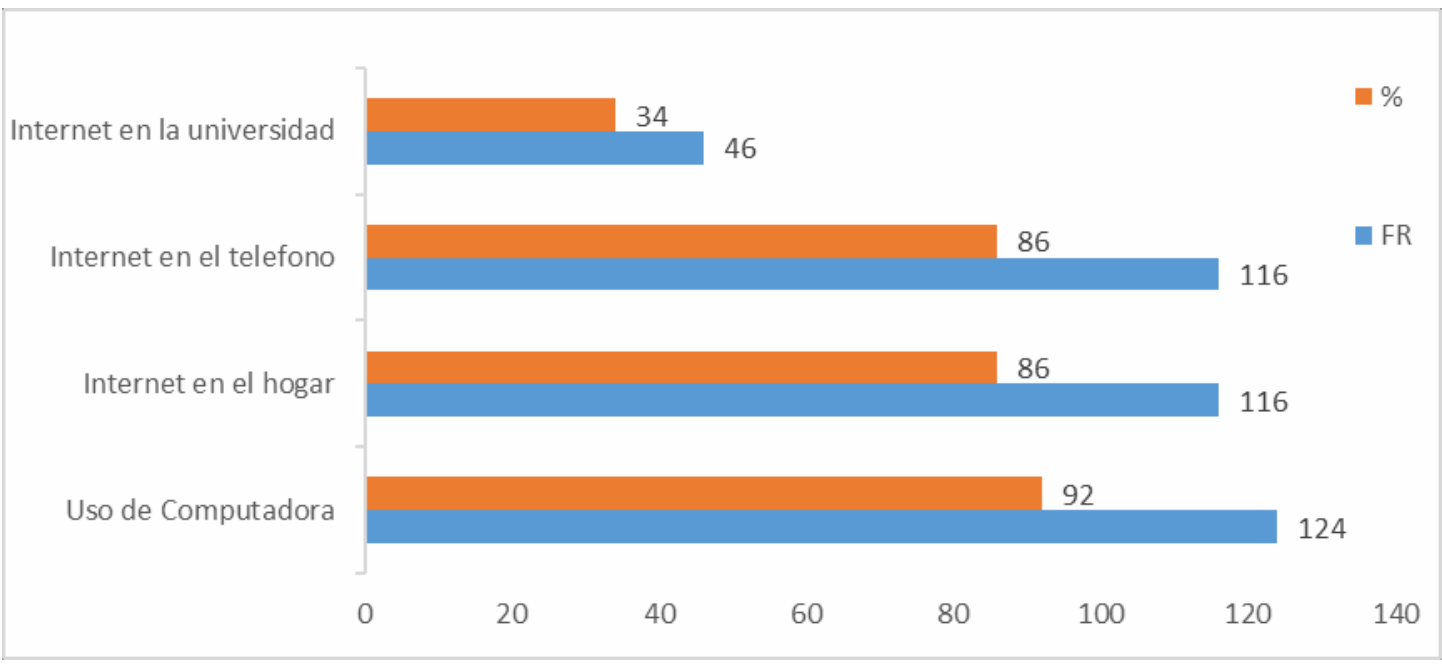

Grafico 1: Análisis descriptivo frecuencial del uso computacional y acceso a internet

Fuente: Elaboración propia (2018). 
En la tabla y grafico 1, se presenta el análisis descriptivo del uso y manejo de medios computacionales, en la misma se indica que el $92 \%$ de los estudiantes utilizan diariamente la computadora para apoyarse en las labores propias de sus estudios. Asimismo, el 86\% indico que tienen acceso a internet en su casa y teléfono celular y un $34 \%$ en la universidad.

Tabla 2

Análisis descriptivo frecuencial del tiempo y sitios de visitas de las TIC's

\begin{tabular}{ccc}
\hline ANALISIS/ ALTERNATIVAS & FR & $\%$ \\
\hline Posee cuenta de correo & 132 & 98 \\
\hline Más de 10 horas de navegación & 117 & 87
\end{tabular}

Fuente: Elaboración propia (2018).

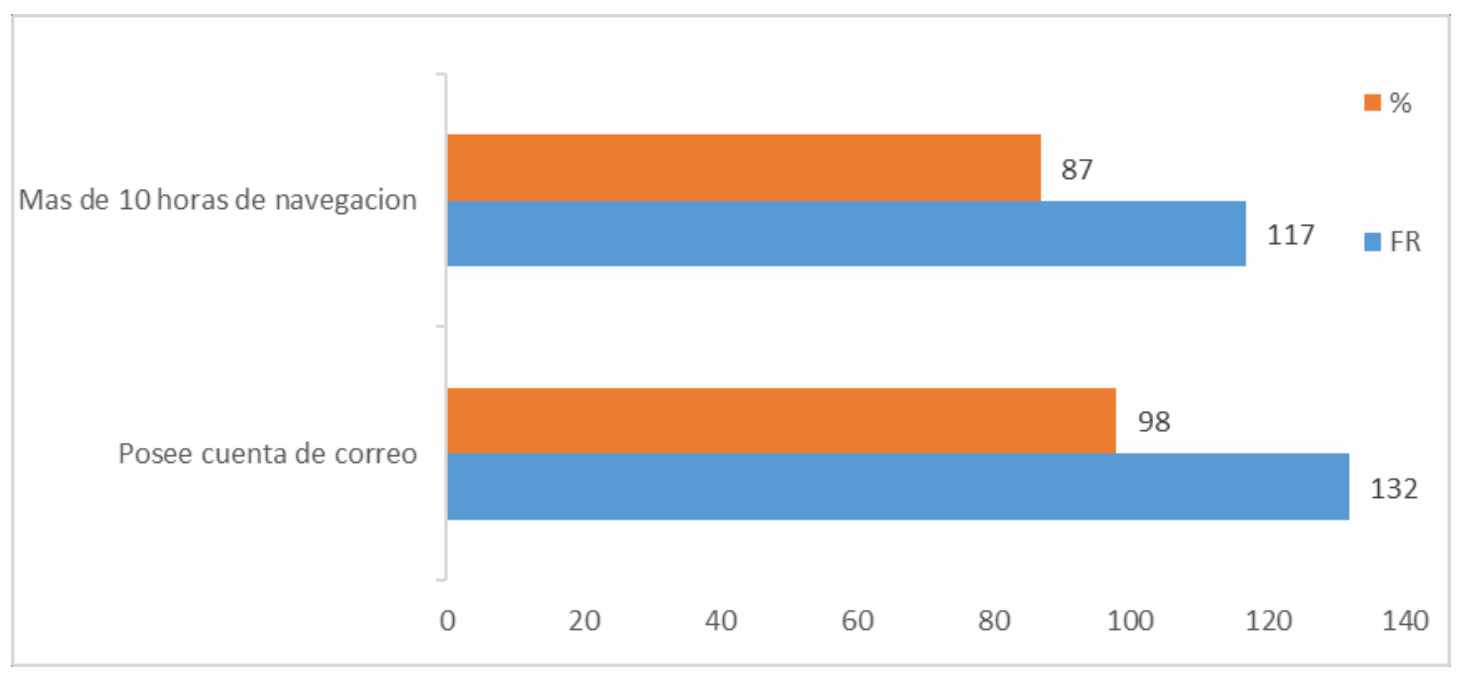

Grafico 2: Análisis descriptivo frecuencial del tiempo y sitios de visitas de las TIC's

Fuente: Elaboración propia (2018). 
En la tabla y grafico 2, se presenta el análisis descriptivo del tiempo y sitios de visitas de las TIC's, en la cual el 98\% de los encuestados coincidieron en que manejan y tienen una cuenta de correo que visitan de forma muy frecuente. Siendo Google el buscador que más utilizan, seguido de Yahoo y Bing, en la cual el 87\% navegan hasta 10 horas o más semanales a través de Internet Explorer, navegador predeterminado en los equipos que cuentan con un sistema operativo Windows.

\section{Tabla 3}

\section{Análisis descriptivo frecuencial del uso y conocimiento de herramientas de internet}

\begin{tabular}{|c|c|c|}
\hline ANALISIS/ ALTERNATIVAS & $F R$ & $\%$ \\
\hline Chat y los blog & 88 & 67 \\
\hline WhatsApp, Twitter, Instagram & 75 & 56 \\
\hline Blogger (Blogspot) y Edublogs. & 39 & 29 \\
\hline
\end{tabular}

Fuente: Elaboración propia (2018).

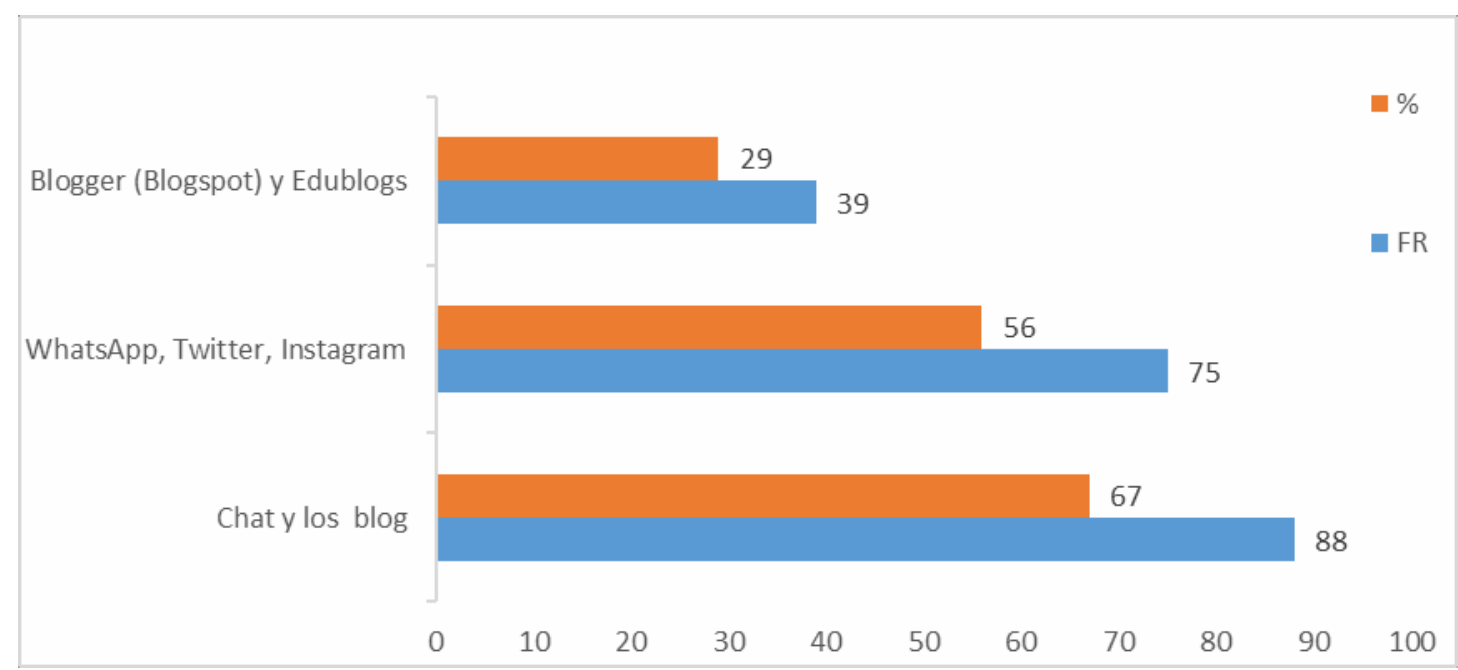

Grafico 3. Análisis descriptivo frecuencial del uso y conocimiento de herramientas de internet

Fuente: Elaboración propia (2018). 
En la tabla y grafico 3, se presenta el análisis descriptivo frecuencial el uso y conocimiento de las herramientas de internet, en la que el $67 \%$ de las opiniones indico que conocen y usan el chat y los blogs. Seguido del $56 \%$ por las redes sociales (WhatsApp, Twitter, Instagram). Con menores porcentajes de uso el Wiki y Foros. Destacándose que solo el 29\% conocen y han utilizados el Blogger (Blogspot) y Edublogs.

\section{Tabla 4}

Análisis descriptivo frecuencial del uso y conocimiento de los programas y sitios de internet

\begin{tabular}{lcc}
\hline \multicolumn{1}{c}{$\boldsymbol{A N A L I S I S / A L T E R N A T I V A S}$} & $\boldsymbol{F R}$ & $\%$ \\
\hline Wikipedia & 118 & 88 \\
\hline Live Messenger & 83 & 62 \\
\hline Yahoo Messenger & 74 & 55 \\
\hline Skype & 58 & 43 \\
\hline Google Maps y You Tube. & 99 & 74
\end{tabular}

Fuente: Elaboración propia (2018).

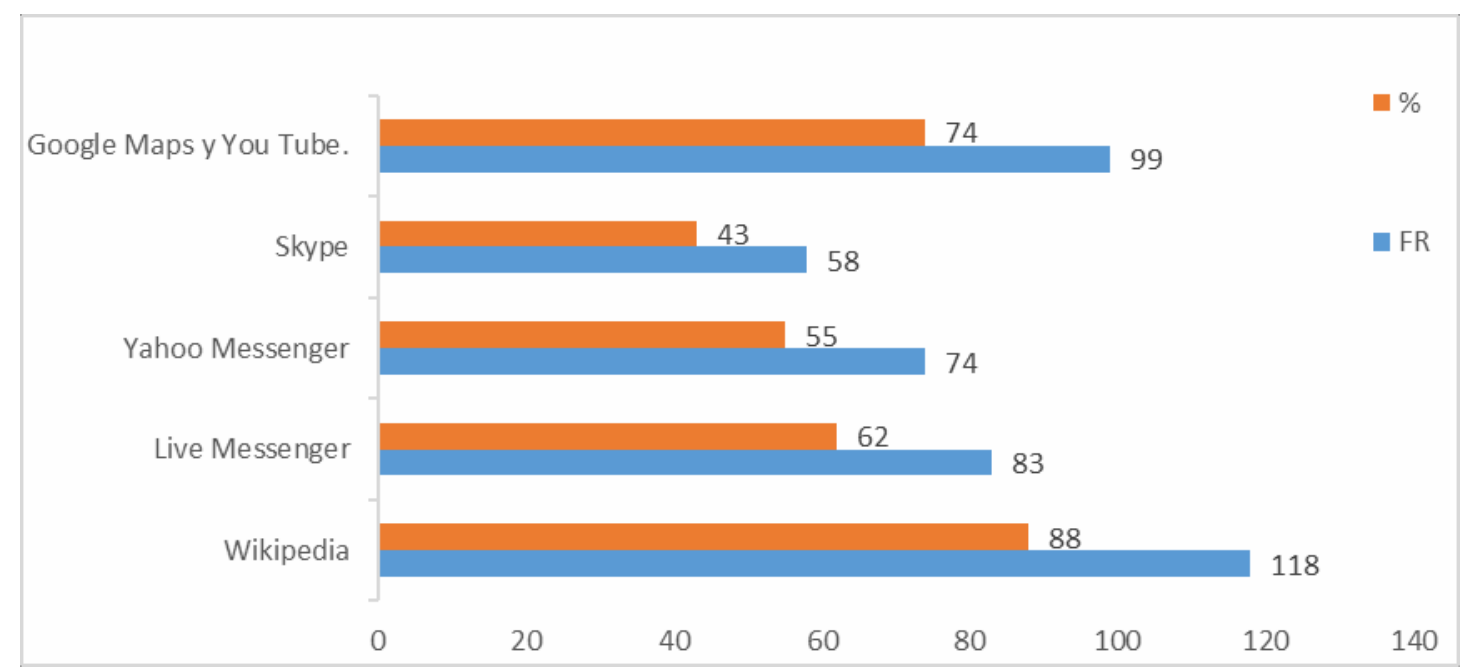


Grafico 4. Análisis descriptivo frecuencial del uso y conocimiento de los programas y sitios de internet Fuente: Elaboración propia (2018).

En la tabla y grafico 4, se presenta el análisis descriptivo frecuencial el uso y conocimiento de los programas y sitios de internet, en la que el $88 \%$ señalo que consultan Wikipedia. Seguido del 62\% que indico Live Messenger (msnmessenger) y el 55\% Yahoo Messenger. El menor porcentaje con el 43\% usa Skype. Entre otros recursos indicaron en un 74\% utilizar Google Maps y You Tube.

\section{Conclusiones}

-Se percibió escaso apoyo tecnológico desde la universidad a sus estudiantes, específicamente en relación al uso del internet para consultar y revisar las actividades propias de su proceso de formación y uso de los correos personales como medio de información académica e interacción de la triada universidad - docente -estudiante.

Existe una alta dedicación horaria de navegación a través de Internet Explorer por parte de los estudiantes, específicamente utilizando el buscador google, seguido del Yahoo y Bing. Siendo las herramientas de internet que más conocen el chat y los blog.

Asimismo, se constató que utilizan Wikipedia como la herramienta de mayor consulta y las redes sociales de WhatsApp, Twitter e Instagram como medio de información y comunicación para informarse de aspectos propios de los contenidos académicos tratados en clases.

\section{Referencias Bibliográficas}

Dussel, I y Quevedo, L. (2010). Educación y nuevas tecnologías: los desafíos pedagógicos ante el mundo digital. Documento básico. Argentina. Fundación Santillana

García Peñalvo, F. (2001). Los espacios virtuales educativos en el ámbito de Internet: Un refuerzo a la formación tradicional, Teoría de la Educación. Educación y Cultura en la Sociedad de la Información. Recuperado en: 
http://www3.usal.es/ teoriaeducacion/rev_numero_03/n3_art_garcia-garcia.htm

Hernández, Fernández, y Baptista, (2014). Metodología de la investigación. Editorial McGraw-Hill. México.

Royero, J. (2007) Redes sociales monografía Elvis J Belial Dìaz Marquis. Recuperado en: $\quad$ http://www.monografias.com/trabajos84/redessociales/redessociales.shtml\#definicioa

Toca, G. (2018). La digitalización no es una maquina digital de precarización masiva.

Revista Ekos. Negocios sostenibles. 297 • Enero 2018 • ekosnegocios.com

UNESCO (2016). La Integración de las tecnologías de la comunicación. Recuperado en: https:/unesdoc.unesco.org/ark:/48223/pf0000150785

Zelaya Medrano, J. (2007) Educación a distancia una alternativa para los Sistemas Educativos. Diseño e impresión: PrintCenter, San José, Costa Rica 